de Mecánica Computacional

Mecánica Computacional Vol XXIX, págs. 9485-9498 (artículo completo)

Eduardo Dvorkin, Marcela Goldschmit, Mario Storti (Eds.)

Buenos Aires, Argentina, 15-18 Noviembre 2010

\title{
OPTIMIZATION OF LAMINATED COMPOSITE STRUCTURES USING HARMONY SEARCH ALGORITHM
}

\author{
Felipe Schaedler de Almeida and Armando Miguel Awruch \\ Graduate Program in Civil Engineering, Federal University of Rio Grande do Sul, Av.Osvaldo Aranha, \\ 99, 90035-190 Porto Alegre, RS, Brazil http://www.ppgec.ufrgs.br/cemacom/
}

Keywords: Laminated composite structures, Optimization, Harmony Search Algorithm

\begin{abstract}
A relatively new heuristic method called Harmony Search (HS) is applied in this work for the optimization of laminated composite structures. This seems to be an unexplored application of the method, although it has already been used for the optimization of frame structures. The Harmony Search algorithm, originally developed to deal with continuous variables, is adapted to handle discrete values allowed for thickness and fiber orientation of the laminate plies, being both taken as design variables. The optimization problem presented in this work is concerned on the minimization of weight and deflection of a laminated composite plate under transverse distributed load. The finite element method is used for the linear analysis of the plate. Ply failure and maximum thickness of contiguous plies with the same fiber orientation are the design constraints. The algorithm performance is evaluated in terms of the apparent reliability (or the chance to find the optimum solution in a single optimization) and computational cost (given in terms of the average number of structural analyses required in a single optimization). Results obtained here showed that the performance of the Harmony Search algorithm was better than the genetic algorithm (GA) for solving the problem studied in this work. This indicates that Harmony Search is a promising method for the optimization of laminated composites.
\end{abstract}




\section{INTRODUCTION}

The outstanding mechanical properties of composite materials have converted these kind of materials in one of the most interesting choices for high performance structural applications. This fact is due to the high specific stiffness and/or high specific strength presented by advanced composites, such as those made of an epoxy matrix reinforced by continuous graphite or aramid fibers. A lamina made of such materials has strong orthotropic behavior with higher stiffness and strength occurring in fiber direction while lower properties occur in transverse directions to fiber orientation.

The use of laminated composites avoids the material weakness in some directions since each ply of the laminate can have a different fiber orientation. Tailoring of laminated composite for a specific application is possible by changing the number of plies and their fiber orientation. The use of right values for these design variables can lead to substantial improvement in the final structural application. However, the determination of the best configuration is usually related to the solution of a huge nonlinear minimization or maximization problem, which is impracticable in usual engineering design process. This is why many research works have been done during the last decades in order to apply optimization algorithms for the design of laminated composite structures.

The first efforts on the optimization of laminated composites were performed using traditional mathematical methods which are based on gradient information of objective and constraint functions in a continuous design space. However, design variables such as the number of plies in the laminate, the thickness and the fiber orientation of the plies are usually limited to discrete values in real structural application. The variable nature and the irregular behavior of objective and constraint functions involved in the optimization of laminated composite make gradient based methods quite inefficient for this application.

Better results were obtained using heuristic methods for the optimization of laminated composites. Some of the most popular methods applied for structural optimization are genetic algorithm (GA), simulated annealing, particle swarm and ant colony, among others, with a lot of papers published. Most of these methods are based on probabilistic procedures that try to mimic the mechanisms of some natural phenomena. In spite of the capacity of these algorithms in finding the optimal or near optimal solution in complex engineering problems, all of them suffer from the drawback of requiring a large number of analyses for each optimization. This issue use to be the bottleneck for the application of the mentioned heuristic methods in problems that use numerical procedures for the analysis of each design configuration, since in this case a large amount of time is required for each objective function evaluation. This means that finding an optimization procedure that require a lower amount of sample evaluations in the design space, allowing the use of high fidelity analysis in the optimization, continues to be a crucial research challenge.

A relatively new heuristic method called Harmony Search (HS) have been developed by Geem et al. (2001) for the optimization of problems with continuous variables. Unlike most of existing heuristic methods, which imitate a natural phenomena, the HS is inspired in the artificial human process of improvisation in music playing in order to search for the perfect sate of harmony. This method creates a new candidate configuration changing design variables in the same way that a musician plays a musical note based on his experience or randomness. The improvement of the musician experience in that harmony is simulated by the continuous improvement of the designs stored in a harmony memory. The objective function has the state of harmony (aesthetic quality) as its counterpart in the analogy between the algorithm and music 
improvisation.

This method have been successfully applied to many engineering problems as demonstrated in Lee and Geem (2005), Kim et al. (2003) and Verma et al. (2010). Harmony Search (HS) algorithms had also been applied in many works dealing with the optimization of skeletal structures as those presented by Lee and Geem (2004), Kang and Jin (2007), Kaveh and Talatahari (2009), Hasanebi et al. (2010). These works have demonstrated that HS may be a very attractive method to solve complex engineering problems with a performance that is expected to be equal or better than that obtained by GA or other popular heuristic methods. In spite of the quality demonstrated by HS, after a careful search the authors could not find any work dealing with the application of this method for optimization of laminated composite structures, which indicates that the present work seems to be the first on this subject.

This work is concerned with the application of Harmony Search algorithm to the optimization of laminated composite structures. Such task is performed exploring three points. The most basic one is to determine if HS can find the optimal solution of a usual problem involving the design of a simple laminated composite structural part. The second point to be studied is the efficiency of HS in such problem. Considering the probabilistic nature of HS, its efficiency is evaluated by measuring the chance to find the optimum solution and the average number of structural analyses required in a single optimization, which is determined by the result of several executions of the algorithm. The last topic to be investigated is the sensitivity of the algorithm efficiency with respect to its parameters.

A problem related to the simultaneous minimization of weight and central deflection of a transversally loaded laminated plate subject to failure constraints is adopted in this work to perform the studies described above. In such multiobjective problem different emphasis may be given to each of the two objectives according to the value of a weighting factor used in the objective function. In the present work three situations of objectives weighting are considered. This problem has been addressed in a previous work using genetic algorithms as the optimization tool (Almeida and Awruch, 2009), which allows a comparison on the efficiency of both methods. Furthermore, all the possible laminated configurations in the design space were analyzed by a finite element code and the necessary information to evaluate the objective function was stored. Using this information enabled the execution of numerous optimizations to evaluate the HS performance.

\section{THE HARMONY SEARCH ALGORITHM}

The algorithm is composed by few simple steps which are briefly described below. A careful explanation for each step is given in Geem et al. (2001).

A design $a$ is represented in the algorithm by a vector $\mathbf{X}^{a}=\left\{X_{1}^{a} X_{2}^{a} X_{3}^{a} \cdots X_{n-1}^{a} X_{n}^{a}\right\}^{T}$ containing the value for each one of the $n$ design variables of the problem. Each design variable may have an integer value varying from 1 to the number of possible admissible discrete values for that variable. The actual values of the variables are linked to the integer numbers in a table which may be consulted before the objective function evaluation takes place. A group of the best designs, called harmony memory (HM), is maintained by the algorithm during the optimization. The worst design in HM is replaced when a better candidate is generated and the number of design kept in the memory (HMS) is one of the parameters of the algorithm.

HS generates new candidate design by defining the value of each variable in three ways. The first way is to randomly choose a value in the admissible range. The second form is to use the variable value taken from a randomly selected design vector in the HM. When the value is taken from the HM it can also have a little modification in a procedure that consists 
in the third way of defining each variable of the new design. The decision on which procedure will be used to obtain the value of a design variable is based on a probabilistic process. The chance to use a value from the memory instead to generate randomly this value is given by the parameter HMCR (harmony memory considering rate). If the value is taken from the memory, then the modification procedure is applied with a probability given by the parameter PAR (pitch adjusting rate). In this work the modified value of the variable $X_{i}$, originally obtained from $\mathrm{HM}$, is taken as a surrounding value $X_{i}=X_{i} \pm 1$, with the sign randomly picked with the same chance. Both HMCR and PAR must have a value between 0.0 and 1.0, and the process defined by each one of this parameters occurs if a random number obtained in the range $(0.0,1.0]$ is lower or equal to them.

An algorithm iteration is formed by the processes of generating one candidate design, evaluating its objective function value and finally updating HM if the new design is better than the worst design stored. This process is repeated until the maximum number of iterations $\left(\mathrm{IT}_{\max }\right)$ is reached or when other end criterion is satisfied.

The HS is based in the following steps:

Step 1 Initialization of optimization problem and the algorithm parameters

Set the values for the algorithm parameters HMS, HMCR, PAR and $\mathrm{IT}_{\max }$. Define the number of variables and the admissible values of each variable. Initialize the objective function evaluation procedure if necessary.

Step 2 Initialization of the Harmony Memory

Fill HM with HMS design vectors randomly generated, respecting the admissible range for each variable.

Step 3 Improvise a new harmony

Generate a new design using the procedure described above and evaluate its objective function.

Step 4 Update Harmony Memory

If the new design is better than the worst design in HM then an update of HM occurs.

Step 5 Repeat steps 3 and 4 until the end criterion is satisfied

A further modification, proposed by Mahdavi et al. (2007), is introduced in the standard algorithm described above. The difference between original and modified HS consists in the use of a variable value of PAR during the optimization. The value of PAR in a iteration $i$ is given by

$$
P A R(i)=P A R_{\min }+i \frac{P A R_{\max }-P A R_{\min }}{\mathrm{IT}_{\max }}
$$

where $P A R_{\min }$ and $P A R_{\max }$ are the minimum and maximum value of PAR which occurs in the beginning and at the end of the optimization process, respectively. These modifications are introduced in order to eliminate the poor efficiency of the algorithm observed in previous works (Mahdavi et al. (2007); Kaveh and Abadi (2010)) when fixed values of PAR were adopted. 


\section{NUMERICAL APPLICATION}

This section presents a study to evaluate the efficiency of HS when applied to the optimization of laminated composite structures. The investigation is based on a single optimization problem described below. Finally, results are presented and discussed.

\subsection{Problem description}

This example, which was previously studied by Almeida and Awruch (2009) using GA, deals with the design of a composite laminated square plate subjected to a uniform pressure load on its surface. Minimization of the structural weight and deflection are the design objectives which must be considered together with constraints on material failure and maximum values of contiguous plies thickness with the same fiber orientation.

A multiobjective approach is adopted for the optimization, since both objectives are simultaneously considered. This is accomplished by using an objective function containing a weighted sum of both objectives. A weighting factor $\alpha$ controls the emphasis given to each one of the objectives in the optimization. Optimizations with different values of $\alpha$ results in a distinct optimum, and consequently, the problem has a set of optimal solutions (pareto-optimal set) instead of a single solution.

The structure geometry, boundary conditions and the mechanical properties of the composite material are presented in Fig. 1. The elastic constants are the Young's modulus in the fiber direction $\left(E_{1}\right)$ and transverse to the fiber direction $\left(E_{2}\right)$, the shear modulus $\left(G_{12}\right)$ and the Poisson's ratio $\left(\nu_{12}\right)$, respectively. Strength parameters for traction and compression for longitudinal and transversal directions are given by $F_{1 t}, F_{1 c}, F_{2 t}$, and $F_{2 c}$, respectively. The remainder parameters are the shear strength $\left(F_{6}\right)$ and the specific weight $(\rho)$. The structure must support a design pressure load of $0.1 \mathrm{MPa}$ with no material failure, which is satisfied when the value of Tsai-Wu failure function (see Jones (1999)) is lower than 1.0 for the whole plate. An additional constraint is imposed in the thickness of contiguous plies with the same fiber orientation, which must not be greater than $2 \mathrm{~mm}$.

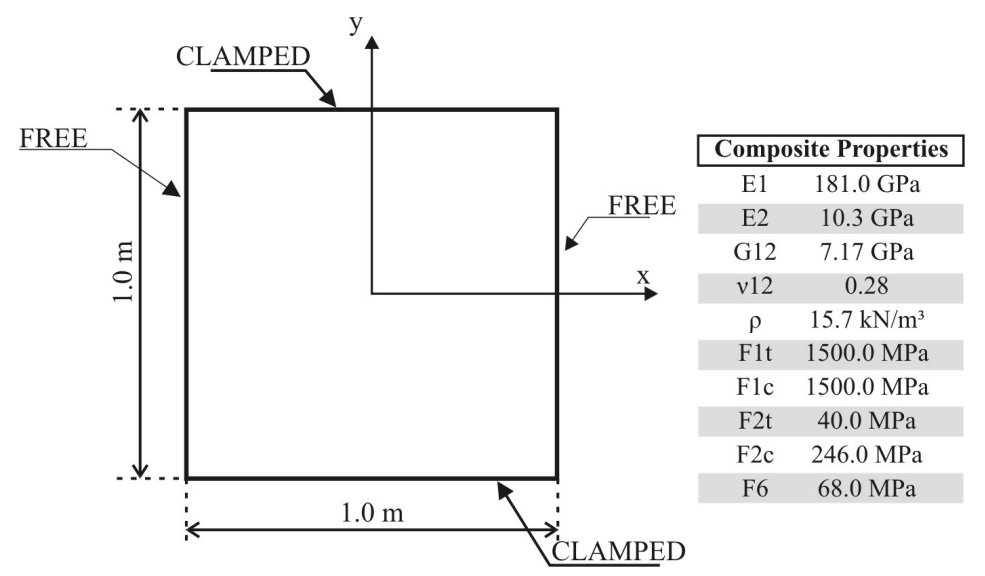

Figure 1: Structure geometry, boundary conditions and composite properties

The laminate is restricted to be symmetric with 8 layers, which means that only 4 layers needs to be represented in the optimization procedure. The fiber orientation angle and the thickness of each layer are the optimization variables, which must have one of the discrete values given in Table 1. A candidate design is represented using 4 variables for the plies angle and 4 variables 
for plies thickness. The number of variables combined with the number of possible values of each variable lead to the size of the design space (SDS), which is equal to 65536.

Table 1: Integer code associated to each possible discrete variable values

\begin{tabular}{cccc}
\hline \multicolumn{2}{c}{ Fiber orientation } & \multicolumn{2}{c}{ Ply thickness } \\
\hline Code & Orientation angle & Code & Ply thickness \\
1 & 1 ply at $0^{\circ}$ & 1 & $0.75 \mathrm{~mm}$ \\
2 & 1 ply at $-45^{\circ}$ & 2 & $1.00 \mathrm{~mm}$ \\
3 & 1 ply at $+45^{\circ}$ & 3 & $1.50 \mathrm{~mm}$ \\
4 & 1 ply at $90^{\circ}$ & 4 & $2.00 \mathrm{~mm}$ \\
\hline
\end{tabular}

The objective function containing the weighted sum of the objectives considered for the plate optimization is given in Eq. (2). The constraints are considered by a penalty formulation of the objective function, where unfeasible designs have a reduction on their fitness proportionally to the magnitude of the constraints violation.

$$
\begin{aligned}
& f_{o b j}=\left(\alpha W^{*}+(1-\alpha) D^{*}\right)(T v+1)^{5}, \quad \text { if } F F \leq 1 \\
& f_{o b j}=\left(\alpha W^{*}+(1-\alpha) D^{*}\right)((T v+1) F F)^{5}, \quad \text { if } F F>1
\end{aligned}
$$

Dimensionless variables $\mathrm{W}^{*}$ and $\mathrm{D}^{*}$ representing the total weight and the central deflection of the plate normalized by their maximum and minimum values are used in the equation. This approach is more efficient than a formulation that uses directly the weight and displacement value or even a simple dimensionless value of these variables divided by a reference value (see Almeida (2006)). The normalization of the variables is given by Eq. (3) and Eq. (4), where the coefficient 1.0 is added to avoid nulls values of $W^{*}$ and $D^{*}$.

$$
\begin{aligned}
W^{*} & =\frac{W-W_{\min }}{W_{\max }-W_{\min }}+1 \\
D^{*} & =\frac{D-D_{\min }}{D_{\max }-D_{\min }}+1
\end{aligned}
$$

The parameters $F F$ and $T v$ are introduced in Eq. (2) to penalize unfeasible designs. The first one represents the maximum value of the failure function evaluated in the structure, with a value greater than 1.0 indicating a failure. The parameter $T v$ is referred to the violation of the limit of contiguous plies thickness with the same fiber orientation, with its value given by the exceeding thickness violating the fixed limit.

The weight and central displacement of all the feasible designs in the problem are shown in Fig. 2. Points $\mathrm{A}$ to $\mathrm{P}$ in this figure are the designs that form the pareto-optimal set, which must be obtained in the optimization depending on the emphasis given to each of the objectives. Details of the pareto-optimal set are presented in Table 2.

\subsection{Investigation on the influence of parameters over algorithm performance}

This section presents the evaluation of HS performance when this algorithm is applied to the problem described above. The sensitivity of HS to its parameters is also investigated. Three 


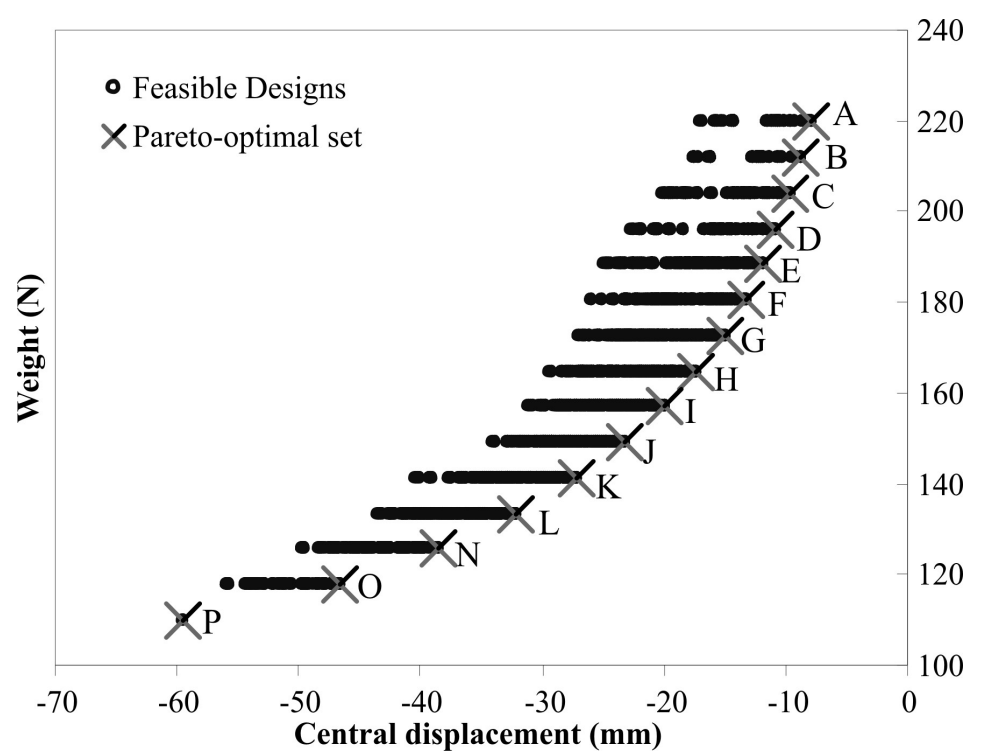

Figure 2: Weight and central displacement of feasible designs

Table 2: Pareto optimal designs

\begin{tabular}{|c|c|c|c|c|}
\hline $\begin{array}{l}\text { Optimal } \\
\text { design }\end{array}$ & Laminate & $\begin{array}{l}\text { Weight } \\
\text { (N) }\end{array}$ & $\begin{array}{l}\text { Deflection } \\
\quad(\mathrm{mm})\end{array}$ & $\begin{array}{l}\text { Weighting factor } \\
\alpha \alpha\end{array}$ \\
\hline A & $\left.90^{2.0}, \quad+45^{2.0}, \quad 90^{2.0}, \quad-45^{1.0}\right]_{S}$ & 219.7 & -7.9 & $0.0-0.20$ \\
\hline B & $\left.90^{2.0}, \quad-45^{2.0}, \quad 90^{2.0}, \quad+45^{0.75}\right]_{S}$ & 211.9 & -8.8 & - \\
\hline $\mathrm{C}$ & {$\left[\begin{array}{llll}90^{2.0}, & -45^{1.75}, & 90^{2.0}, & +45^{1.0}\end{array}\right]_{S}$} & 204 & -9.6 & 0.25 \\
\hline $\mathrm{D}$ & {$\left[\begin{array}{llll}90^{2.0} & -45^{1.75}, & 90^{2.0}, & +45^{0.75}\end{array}\right]_{S}$} & 196.2 & -10.8 & - \\
\hline $\mathrm{E}$ & {$\left[\begin{array}{llll}90^{2.0}, & -45^{1.0}, & 90^{2.0}, & +45^{1.0}\end{array}\right]_{S}$} & 188.4 & -11.8 & 0.3 \\
\hline $\mathrm{F}$ & {$\left[\begin{array}{llll}90^{2.0}, & -45^{0.75}, & 90^{2.0}, & +45^{1.0}\end{array}\right]_{S}$} & 180.5 & -13.2 & 0.35 \\
\hline G & {$\left[\begin{array}{llll}90^{2.0} & -45^{0.75}, & 90^{2.0}, & +45^{0.75}\end{array}\right]_{S}$} & 172.7 & -15 & 0.4 \\
\hline $\mathrm{H}$ & {$\left[\begin{array}{llll}90^{2.0}, & -45^{0.75}, & 90^{1.75}, \quad+45^{1.0}\end{array}\right]_{S}$} & 164.8 & -17.3 & 0.45 \\
\hline I & $\left.90^{2.0}, \quad-45^{0.75}, \quad 90^{1.75}, \quad+45^{0.75}\right]_{S}$ & 157 & -19.9 & 0.5 \\
\hline $\mathrm{J}$ & {$\left[\begin{array}{llll}90^{2.0}, & -45^{0.75}, & 90^{1.0}, & +45^{1.0}\end{array}\right]_{S}$} & 149.1 & -23.3 & 0.55 \\
\hline $\mathrm{L}$ & {$\left[\begin{array}{llll}90^{2.0}, & -45^{0.75}, & 90^{1.0}, & +45^{0.75}\end{array}\right]_{S}$} & 141.3 & -27.2 & 0.6 \\
\hline M & $\left.90^{2.0}, \quad-45^{0.75}, \quad 90^{0.75}, \quad-45^{0.75}\right]_{S}$ & 133.4 & -32.1 & 0.65 \\
\hline $\mathrm{N}$ & {$\left[\begin{array}{lll}90_{2}^{1.0}, & -45^{1.0}, & 90^{1.0}\end{array}\right]_{S}$} & 125.6 & -38.5 & 0.7 \\
\hline $\mathrm{O}$ & {$\left[\begin{array}{lll}90_{2}^{1.0}, & +45^{0.75}, & -45^{1.0}\end{array}\right]_{S}$} & 117.7 & -46.6 & $0.75-0.85$ \\
\hline $\mathrm{P}$ & {$\left[90_{2}^{1.0}, \quad 0^{0.75}, \quad+45^{0.75}\right]_{S}$} & 109.9 & -59.5 & $0.90-1.0$ \\
\hline
\end{tabular}


values are considered for the parameters HMS, HMCR, PAR ${ }_{\text {min }}$ and PAR $\mathrm{Pax}_{\text {max }}$, as given in Table 3. Three cases of the multiobjective optimization are considered using values of $\alpha$ equal to 0.25 , 0.50 and 0.75 .

Table 3: HS parameters values

\begin{tabular}{ccccc}
\hline HS parameter & & value 1 & value 2 & value 3 \\
\hline HMS & $=$ & 10 & 20 & 30 \\
HMCR & $=$ & 0.70 & 0.80 & 0.90 \\
PAR_min & $=$ & 0.20 & 0.30 & 0.40 \\
PAR_max & $=$ & 0.70 & 0.80 & 0.90 \\
\hline
\end{tabular}

The algorithm performance is evaluated in terms of two parameters. The first one is the apparent reliability $(\mathrm{R})$, which is determined by taking the number of successful optimizations in finding the global optimum $(\mathrm{No})$, divided by the total number of performed optimizations $(N)$. This quantity defines the chances of obtaining the global optimum in a single optimization process. The second parameter used to evaluate HS performance is the number of analyses (An) required in a single optimization. The computational cost of the algorithm is given by this parameter because the analysis of a possible candidate design using a high fidelity numerical method is the most consuming task in the process. The analysis of a single design is counted only once since analyses results can be stored and used later if the same point (i.e. a possible candidate design) occurs in the optimization.

Table 4 shows the reliability and average number of analyses found by Almeida and Awruch (2009) when solving the problem discussed here using a GA adapted with special operators for the specific case of laminated composite optimization.

Table 4: Genetic Algorithm performance for the present problem

\begin{tabular}{cccc}
\hline$\alpha$ & Optimal designs & $\mathrm{R}$ & $\mathrm{An}$ \\
\hline 0.25 & $\mathrm{C}$ & 0.98 & 4645 \\
0.5 & $\mathrm{I}$ & 1.00 & 4392 \\
0.75 & $\mathrm{O}$ & 0.94 & 3568 \\
\hline
\end{tabular}

HS performance is defined using the results of 75 optimizations performed for each combination of the parameters given in Table 3. All optimization were stopped after 2500 iterations. The results are presented in Fig. 3, Fig. 4 and Fig. 5 for optimizations considering $\alpha$ equal to $0.25,0.50$ and 0.75 , respectively. The optimal solution for these values of $\alpha$ are given by the design points $\mathrm{C}$, I and $\mathrm{O}$ shown in Table 2. Combinations of $\mathrm{PAR}_{\min }$ and $\mathrm{PAR}_{\text {max }}$, defined in Table 5, are used in order to favor results presentation.

Results obtained in the investigation presented in this section show that HS can find the best design in laminated composite optimization problems. However, the algorithm parameters have strong influence in the reliability and computational cost of the optimizations. Good results were obtained for the optimizations with $\alpha$ equal to 0.25 and 0.50 , while poor reliability was obtained for the optimizations with $\alpha=0.75$. This fact may be associated with the modification of objective function behavior as $\alpha$ increases. It can be seen from Eq. (2) that $\alpha$ dictates the 
Table 5: Combinations of $\mathrm{PAR}_{\min }$ and $\mathrm{PAR}_{\max }$

\begin{tabular}{ccccccccc}
\hline Comb. & PAR $_{\text {min }}$ & PAR $_{\text {max }}$ & Comb. & PAR $_{\text {min }}$ & PAR $_{\max }$ & Comb. & PAR $_{\text {min }}$ & PAR $_{\text {max }}$ \\
\hline 1 & 0.2 & 0.7 & 4 & 0.3 & 0.7 & 7 & 0.4 & 0.7 \\
2 & 0.2 & 0.8 & 5 & 0.3 & 0.8 & 8 & 0.4 & 0.8 \\
3 & 0.2 & 0.9 & 6 & 0.3 & 0.9 & 9 & 0.4 & 0.9 \\
\hline
\end{tabular}
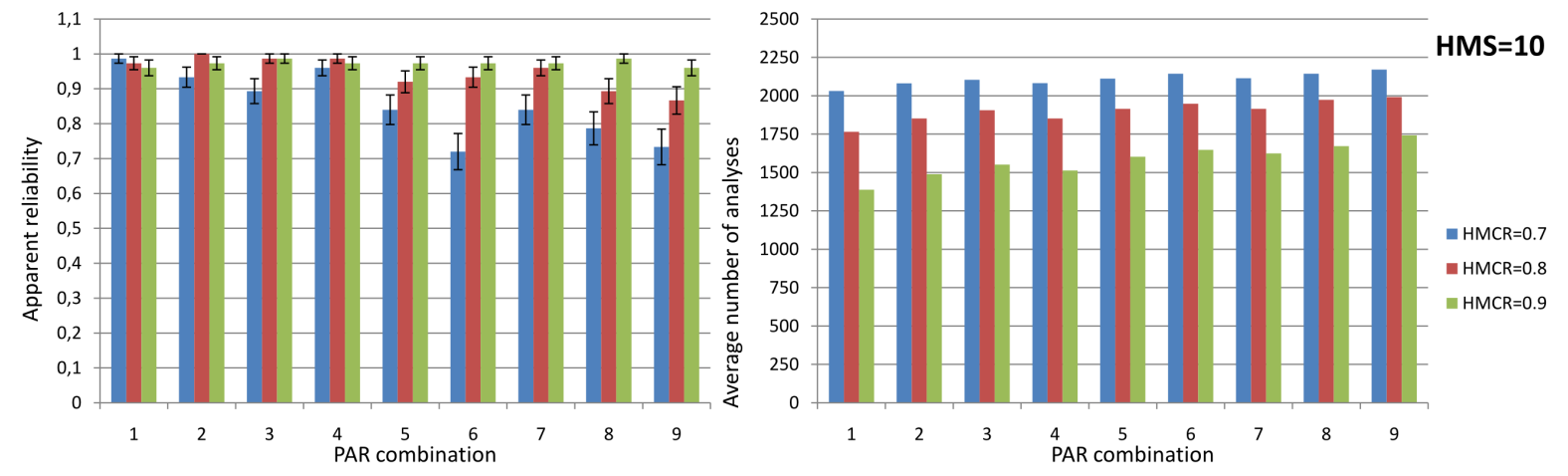

(a) $\mathrm{HMS}=10$
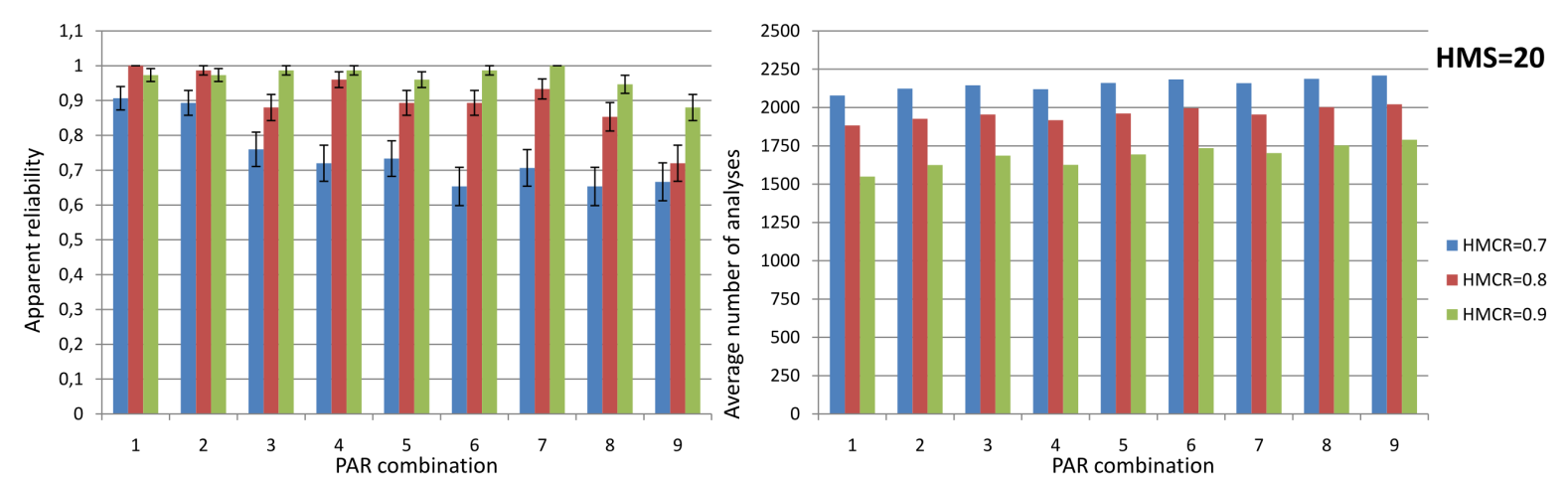

(b) HMS $=20$
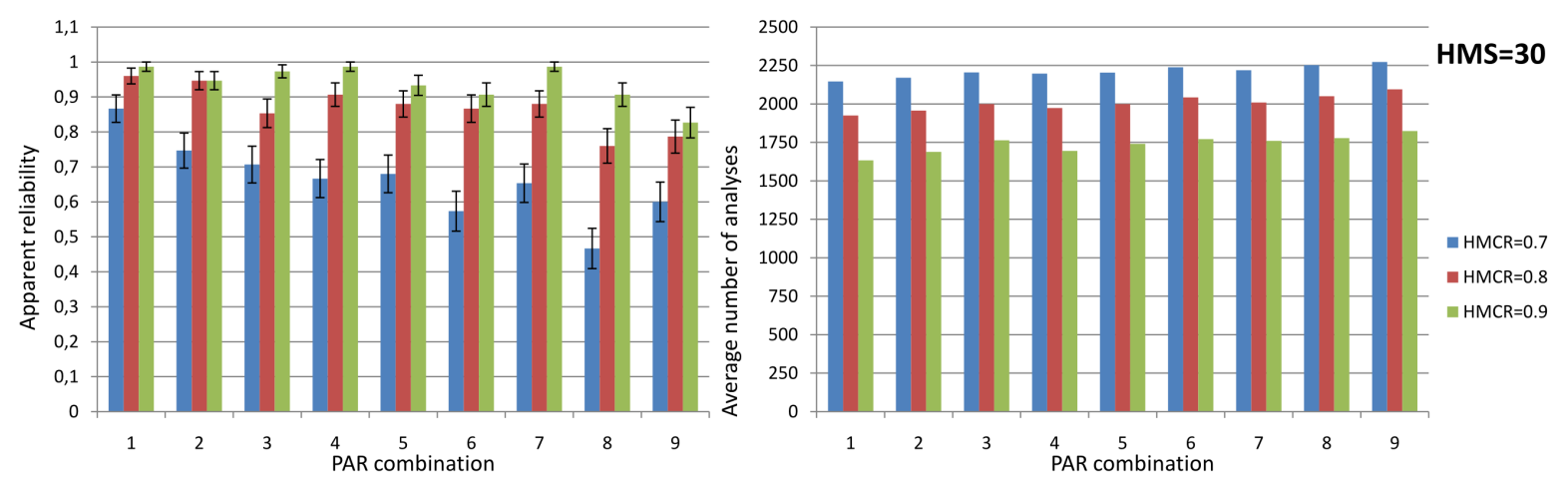

(c) $\mathrm{HMS}=30$

Figure 3: Apparent reliability and average number of analyses for optimizations with $\alpha=0.25$

importance given to plate weight in the optimization. When $\alpha$ is bigger, more importance is given to the weight and less importance is given to the deflection. However, as is shown by the horizontal lines formed by feasible points in Fig. 2, there are a large number of designs with the 

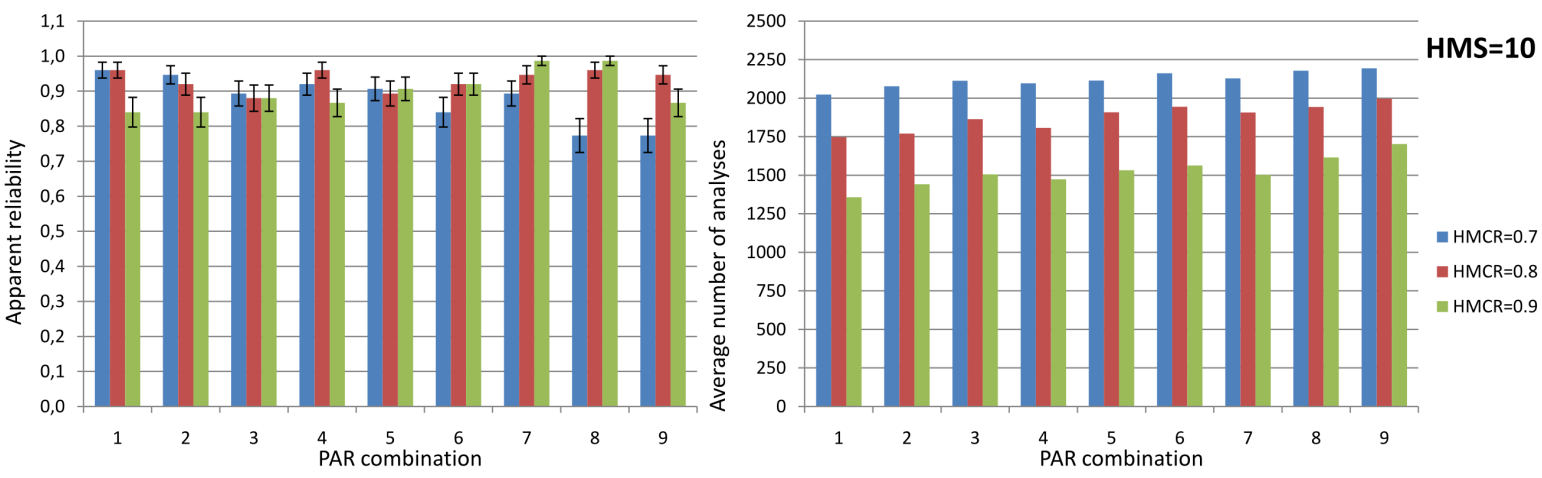

(a) $\mathrm{HMS}=10$
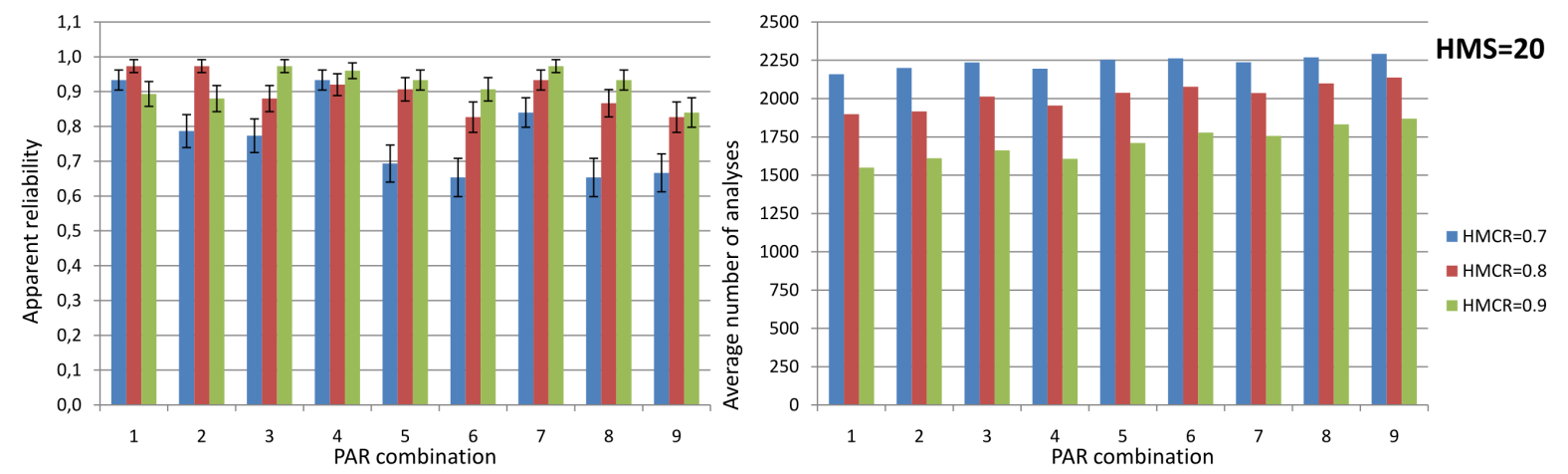

(b) $\mathrm{HMS}=20$
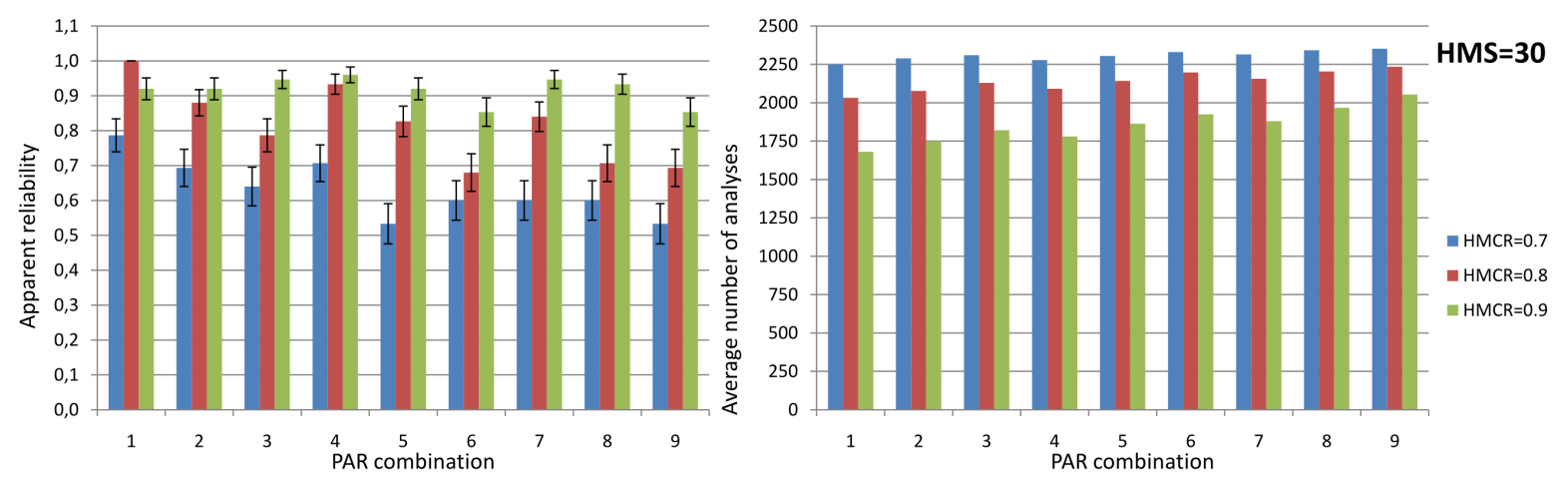

(c) $\mathrm{HMS}=30$

Figure 4: Apparent reliability and average number of analyses for optimizations with $\alpha=0.50$

same weight and small difference in the deflection. This means that the problem with $\alpha$ equal to 0.75 has much more local quasi optimum points than the problems where $\alpha$ equal to 0.25 and 0.50 are used, resulting in a much more difficult optimization process. This indicates that a different setting of HS parameters and more iterations are necessary to perform the optimization when $\alpha=0.75$. An additional investigation on this case is presented in the following sections, while discussions in this section are concerned on the results obtained for the optimization with lower values of $\alpha(\alpha=0.25$ and $\alpha=0.50)$.

Some tendencies on the influence of the parameters studied in this section over the algorithm efficiency can be obtained from Fig. 3 and Fig. 4. Higher values of HMCR clearly decrease the average number of analyses (An) in the optimization and also increase the reliability (R) in most cases. The number of design vectors in memory (HMS) demonstrate a weaker influence 

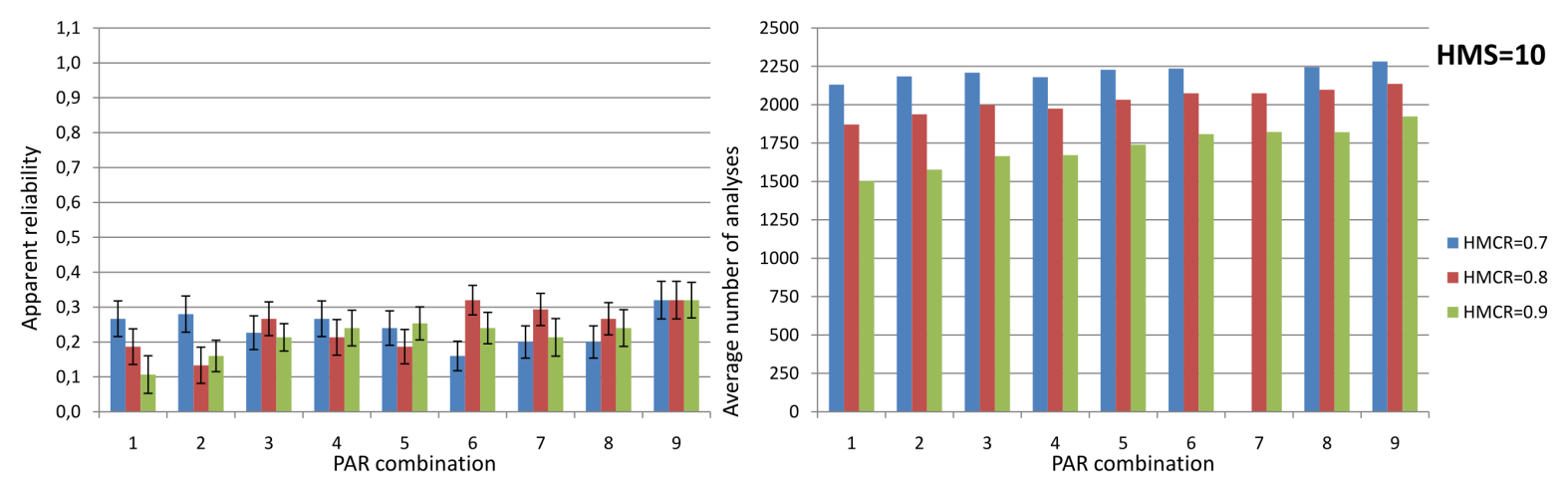

(a) $\mathrm{HMS}=10$
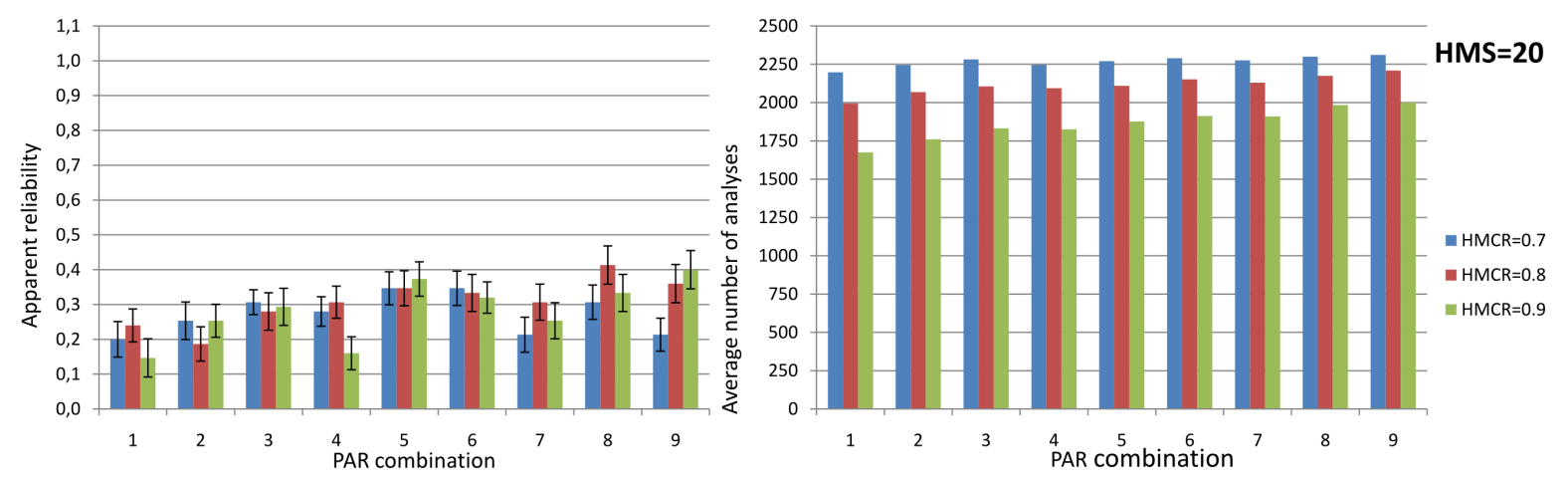

(b) $\mathrm{HMS}=20$
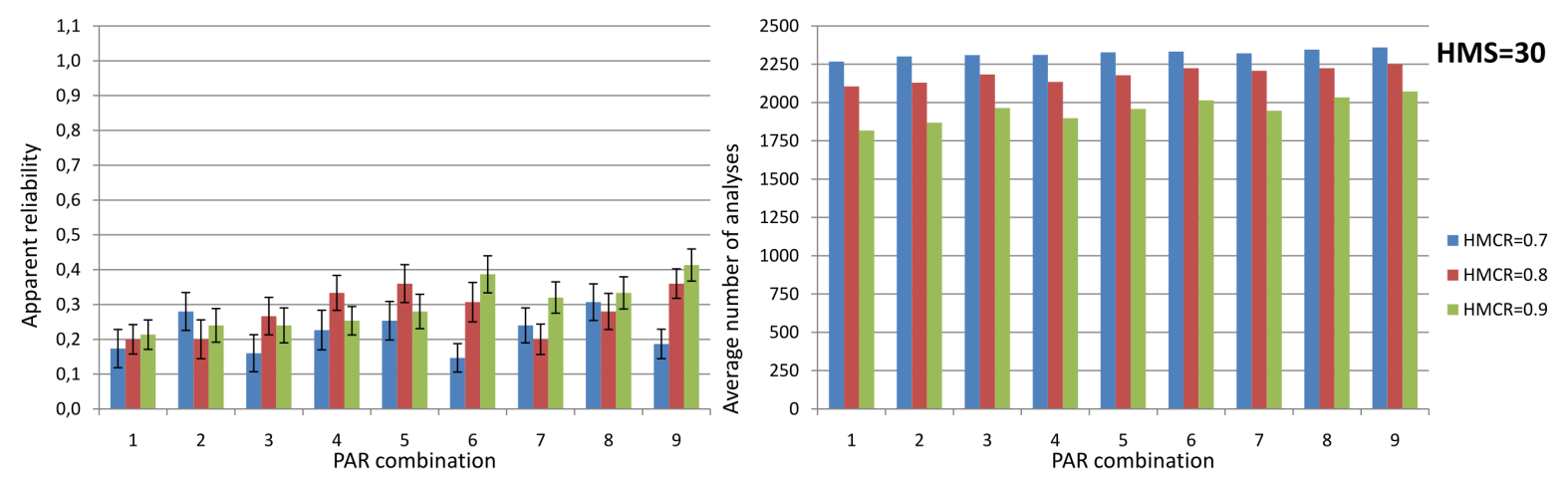

(c) $\mathrm{HMS}=30$

Figure 5: Apparent reliability and average number of analyses for optimizations with $\alpha=0.75$

in HS efficiency. Nevertheless, a larger An and lower R are observed when HMS is bigger. Setting lower values of $\mathrm{PAR}_{\text {min }}$ lead to lower values of An and improve $\mathrm{R}$. This behavior is also observed for the parameter $\mathrm{PAR}_{\max }$, but with less intensity.

A good reliability level ( $\mathrm{R}>0.90)$ was obtained for many parameter combinations. Optimizations with HS were performed requiring usually less than a half of the number of structural analyses needed in the optimizations using genetic algorithm (see Table 4).

\subsection{Adequate HS parameters for optimization using $\alpha=0.75$}

The range of values used for the HS parameters HMS, HMCR and $\mathrm{PAR}_{\min }$ and $\mathrm{PAR}_{\max }$ in the investigations presented above are clearly not adequate for the optimization when $\alpha=$ 0.75 is considered. Further optimizations were performed in order to determine the adequate 
HS parameters in this case. As expected, these results demonstrated that a larger number of iterations are necessary in order to obtain optimizations with good reliability. The tendency to obtain better results when higher values of HMCR are used was also observed. On the other hand, the parameters HMS, $\mathrm{PAR}_{\text {min }}$ and $\mathrm{PAR}_{\max }$ showed to have an influence with opposite effects than those observed in previous results for optimizations using $\alpha$ equal to 0.25 and 0.50 . Adequate reliability levels were obtained only for optimizations using the higher values of these parameters. Additionally, adequate setting of HMS showed to be a key aspect for the success of the optimization.

The behavior described here is illustrated in Fig. 6, where the reliability and the average number of analyses are presented for optimizations with different values of HMS and $\mathrm{IT}_{\max }$. The algorithm performance was evaluated based on the results of 50 optimization performed for each combination of the HS parameters. In this optimizations the following fixed values were used: $\mathrm{HMCR}=0.90, \mathrm{PAR}_{\min }=0.40$ and $\mathrm{PAR}_{\max }=0.90$ (equivalent to PAR combination 9 in Table 5). Values equal to 10, 20, 30, 50 and 80 were used for HMS, while the maximum number of iterations were 7500, 10000 and 12500.
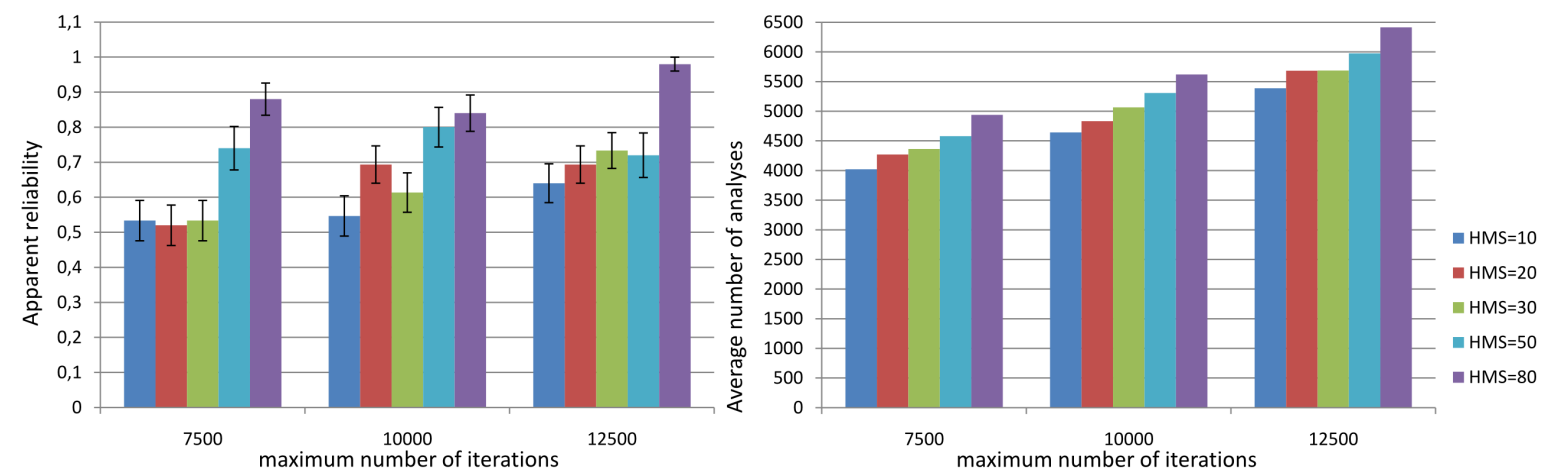

Figure 6: Alternative results for apparent reliability and average number of analyses for optimizations with $\alpha=0.75$ using adequate parameters

The significant improvement in reliability levels obtained with the new adjustments of the HS parameters has been accompanied by a strong increase in the number of analyses required. For similar levels of reliability, HS required almost twice the number of analyses demanded by GA.

\section{CONCLUSIONS}

A study on the application of the Harmony Search algorithm for the optimization of laminated composite structures was presented in this paper. The algorithm efficiency was investigated based on the results obtained for a multiobjective problem, which considers an objective function containing the weighted sum of two objectives and a penalty due to constraints violations. Three values of the weighting parameter used in the objective function were analyzed, resulting in optimizations with different emphasis on each objective and different difficulties for the algorithm were found. The apparent reliability and the average number of structural analyses required in a single optimization were the parameter used to determine HS efficiency in dealing with the proposed problem. The sensitivity of the algorithm with respect to its parameter values was also investigated.

Distinct behaviors of the algorithm were observed for the optimizations according to the value of the weighting parameter $(\alpha)$ in the objective function. HS demonstrates a very good 
efficiency in solving the optimizations when the lower and the intermediate value of $\alpha$ were considered. In these cases high levels of reliability (R) were obtained for many parameter combinations together with a moderate number of analyses required for a single optimization (An). Lower values adopted for the HS parameters HMS, PAR ${ }_{\text {min }}$ and $\mathrm{PAR}_{\text {max }}$ lead to higher $\mathrm{R}$ and lower An.

The optimization problem considering the highest value of $\alpha$ revealed to be much more difficult to solve using HS than the other cases. Poor reliability was obtained in optimizations performed with HS parameters in the range adopted for the study with lower values of $\alpha$ ( $\alpha=$ 0.25 and $\alpha=0.50)$ presented above. In a further investigation it was found that more iterations were necessary in order to obtain a good reliability level for the algorithm in this problem. The influence of the HS parameters HMS, PAR ${ }_{\text {min }}$ and $\mathrm{PAR}_{\max }$ showed to be opposite to that observed for the problems with $\alpha=0.25$ and $\alpha=0.50$. In this case better results were obtained when high values of these parameters were used. The parameter HMS, which controls the number of the more suitable designs stored in a memory available during the optimization, was critical for the success of the optimizations.

In both situations, improved results were obtained when the parameter HMCR with the highest value considered in this study was used. HS showed to be a good tool for the optimization of laminated composites. In many cases the computational cost obtained here was lower than the computational cost of optimizations using genetic algorithms. On the other hand, HS showed some difficulties in solving the last optimization problem (with $\alpha=0.75$ ), which is characterized by the existence of many points with almost the same objective function. In this case acceptable levels of reliability were obtained only with a high number of analyses per optimization, which make the general purpose HS used in this work less efficient than the GA with special features for optimization of laminated composites used in the reference work.

\section{ACKNOWLEDGEMENTS}

The authors wish to thank the Brazilian agencies CNPq and CAPES for their financial support.

\section{REFERENCES}

Almeida F. and Awruch A. Design optimization of composite laminated structures using genetic algorithms and finite element analysis. Composite Structures, 88(3):443 - 454, 2009.

Almeida F.S. Otimização de Estruturas de Materiais Compósitos Laminados utilizando Algoritmos Genétcos. Master's Thesis, Programa de Pós-Graduação em Engenharia Civil Universidade Federal do Rio Grande do Sul (PPGEC-UFRGS), 2006.

Geem Z., Kim J., and Loganathan G. A new heuristic optimization algorithm: Harmony search. Simulation, 76(2):60-68, 2001.

Hasanebi O., Erdal F., and Saka M. Adaptive harmony search method for structural optimization. Journal of Structural Engineering, 136(4):419-431, 2010.

Jones R.M. Mechanics of Composite Materials. Taylor \& Francis, 2nd edition, 1999.

Kang S. and Jin K. Configuration optimization of truss structures using harmony search heuristic algorithm. Advanced Materials Research, 24-25:793-796, 2007.

Kaveh A. and Abadi A.S.M. Cost optimization of a composite floor system using an improved harmony search algorithm. Journal of Constructional Steel Research, 66(5):664 - 669, 2010.

Kaveh A. and Talatahari S. Particle swarm optimizer, ant colony strategy and harmony search scheme hybridized for optimization of truss structures. Computers \& Structures, 87(5-6):267 
$-283,2009$.

Kim J.H., Baek C.W., and Kim E.S. Harmony search algorithm: Application to optimal planning of pipe network rehabilitation. pages 147-152. 2003.

Lee K. and Geem Z. A new meta-heuristic algorithm for continuous engineering optimization: Harmony search theory and practice. Computer Methods in Applied Mechanics and Engineering, 194(36-38):3902-3933, 2005.

Lee K.S. and Geem Z.W. A new structural optimization method based on the harmony search algorithm. Computers \& Structures, 82(9-10):781 - 798, 2004.

Mahdavi M., Fesanghary M., and Damangir E. An improved harmony search algorithm for solving optimization problems. Applied Mathematics and Computation, 188(2):1567 - 1579, 2007.

Verma A., Panigrahi B., and Bijwe P. Harmony search algorithm for transmission network expansion planning. IET Generation, Transmission and Distribution, 4(6):663-673, 2010. 